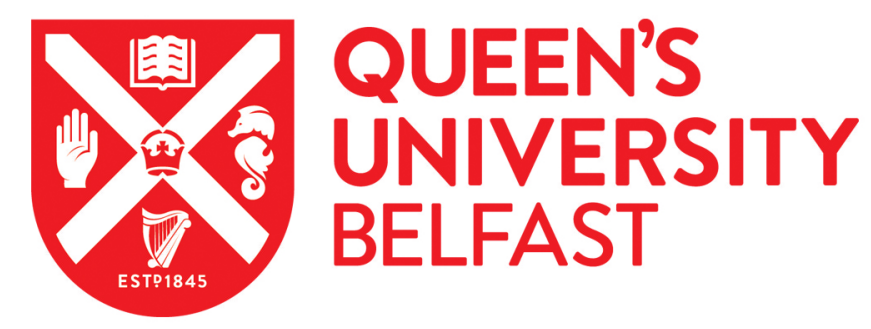

\title{
Vector bundles on the projective line and finite domination of chain complexes
}

Huettemann, T. (2015). Vector bundles on the projective line and finite domination of chain complexes. Mathematical Proceedings of the Royal Irish Academy, 115A(1). https://doi.org/10.3318/pria.2015.115.2

Published in:

Mathematical Proceedings of the Royal Irish Academy

Document Version:

Peer reviewed version

Queen's University Belfast - Research Portal:

Link to publication record in Queen's University Belfast Research Portal

Publisher rights

Copyright 2015 Royal Irish Academy

\section{General rights}

Copyright for the publications made accessible via the Queen's University Belfast Research Portal is retained by the author(s) and / or other copyright owners and it is a condition of accessing these publications that users recognise and abide by the legal requirements associated with these rights.

Take down policy

The Research Portal is Queen's institutional repository that provides access to Queen's research output. Every effort has been made to ensure that content in the Research Portal does not infringe any person's rights, or applicable UK laws. If you discover content in the Research Portal that you believe breaches copyright or violates any law, please contact openaccess@qub.ac.uk. 


\title{
VECTOR BUNDLES ON THE PROJECTIVE LINE AND FINITE DOMINATION OF CHAIN COMPLEXES
}

\author{
THOMAS HÜTTEMANN
}

\begin{abstract}
We present an algebro-geometric approach to a theorem on finite domination of chain complexes over a LAURENT polynomial ring. The approach uses extension of chain complexes to sheaves on the projective line, which is governed by a $K$-theoretical obstruction.
\end{abstract}

Let $R$ be a ring with unit, and let $R((x))=R \llbracket x \rrbracket[1 / x]$ and $R\left(\left(x^{-1}\right)\right)=$ $R \llbracket x^{-1} \rrbracket\left[1 / x^{-1}\right]$ be the rings of formal LAURENT series (finite to the left and right, respectively).

Theorem A (RANicki [Ran95, Theorem 2]). Let $C$ be a bounded complex of finitely generated free $R\left[x, x^{-1}\right]$-modules. The complex $C$ is $R$-finitely dominated (i.e., is a retract up to homotopy, as an $R$-module complex, of a bounded complex of finitely generated free $R$-modules) if both

$$
C \otimes_{R\left[x, x^{-1}\right]} R((x)) \quad \text { and } \quad C \otimes_{R\left[x, x^{-1}\right]} R\left(\left(x^{-1}\right)\right)
$$

are acyclic (and hence contractible) complexes.

(The converse holds as well as shown in [Ran95], see also [Hüt11, HQ13].) The present paper discusses two aspects of this result: An algebro-geometric re-interpretation of the proof given by RANICKI, and an analysis of the "freeness" hypothesis. The latter discussion itself is motivated by the observation that the theorem as given does not allow for iterative application to treat the case of LAURENT polynomial rings $R\left[x, x^{-1}, y, y^{-1}\right]=R\left[y, y^{-1}\right]\left[x, x^{-1}\right]$ in two indeterminates. Indeed, after one application (using $R\left[y, y^{-1}\right]$ in place of $R$ ) we are left with a complex of projective modules rather than free ones. This is relevant as there is a $K$-theoretical obstruction to extending chain complexes to "sheaves on the projective line", which prevents a direct application of the original proof to non-free chain complexes. More precisely, we will prove:

Theorem B. Let $C$ be a bounded chain complex of finitely generated projective $R\left[x, x^{-1}\right]$-modules. The following three statements are equivalent:

(1) The complex $C$ extends to $\mathbb{P}^{1}$ up to quasi-isomorphism.

(2) The class $[C] \in K_{0}\left(R\left[x, x^{-1}\right]\right)$ lies in the image of the map

$$
K_{0}(R) \longrightarrow K_{0}\left(R\left[x, x^{-1}\right]\right), \quad[M] \mapsto\left[M \otimes_{R} R\left[x, x^{-1}\right]\right] .
$$

Date: 24 October 2014.

2010 Mathematics Subject Classification. Primary 18G35; Secondary 55U15, 16E20.

Key words and phrases. Perfect complex; finite domination; $K$-theory; projective line; extension of vector bundle.

This work was supported by the Engineering and Physical Sciences Research Council [grant number EP/H018743/1]. The idea to write this paper was born during a research visit to Academia Sinica in July 2013; their support is gratefully acknowledged. 
(3) The class $[C] \in K_{0}\left(R\left[x, x^{-1}\right]\right)$ lies in the image of the map

$$
i^{*}: K_{0}\left(\mathbb{P}^{1}\right) \longrightarrow K_{0}\left(R\left[x, x^{-1}\right]\right), \quad\left[C^{-} \longrightarrow C \longleftarrow C^{+}\right] \mapsto[C] .
$$

(Here $\mathbb{P}^{1}$ is the projective line over $R$, defined in $\S 4$.) The resulting obstruction can be expressed as an element of a quotient of $K_{0}\left(R\left[x, x^{-1}\right]\right)$ isomorphic to $K_{-1}(R) \oplus N K_{0}(R) \oplus N K_{0}(R)$, and is non-zero in general.

\section{Conventions, And Rules of the game}

Throughout we let $R$ and $S$ denote rings with unit. Modules are right modules. Our complexes are homologically indexed: The differential of a chain complex decreases the degree. If unspecified, complexes are allowed to be unbounded both above and below.

In algebraic geometry, the category of quasi-coherent $\mathcal{O}_{\text {Spec }(S)}$-modules on the affine scheme $\operatorname{Spec}(S)$ is equivalent, via the global sections functor, to the category of $S$-modules. We use this equivalence as a convenient language in the case of non-commutative rings as well. For example, irrespective of commutativity, we say that

- an $S$-module is a quasi-coherent sheaf on $\operatorname{Spec}(S)$;

- $T=\operatorname{Spec} R\left[x, x^{-1}\right]$ is the algebraic torus of dimension 1 over $R$;

- $P=\operatorname{Spec} R$ is the point over $R$;

- the canonical map $p: T \longrightarrow P$ induces a push-forward functor $p_{*}$ which assigns to each quasi-coherent sheaf $M$ on $T$ a quasi-coherent sheaf $p_{*} M$ on $P$, and a pull-back functor $p^{*}$.

The last point says, in module theoretic terms, that an $R\left[x, x^{-1}\right]$-module $M$ can be considered as an $R$-module $p_{*} M$ by restriction of scalars, and that an $R$-module $N$ gives rise to an induced $R\left[x, x^{-1}\right]$-module given by $p^{*} N=$ $N \otimes_{R} R\left[x, x^{-1}\right]$.

\section{Finite domination}

Definition 2.1. A chain complex $C$ of $S$-modules, or quasi-coherent sheaves on $\operatorname{Spec}(S)$, is called

(1) a strict perfect complex if it is bounded and consists of finitely generated projective $S$-modules;

(2) an $S$-perfect complex if it is quasi-isomorphic to a strict perfect complex;

(3) a complex of vector bundles on Spec $S$ if it consists of finitely generated projective $S$-modules;

(4) a complex of trivial vector bundles on $\operatorname{Spec} S$ if it consists of finitely generated free $S$-modules;

(5) an $S$-finitely dominated complex if there exist a bounded complex $D$ of finitely generated free $S$-modules and chain maps $r: D \longrightarrow C$ and $s: C \longrightarrow D$ such that $r \circ s \simeq \operatorname{id}_{C}$.

We will need the following trivial observation: Every strict perfect complex $C$ over $\operatorname{Spec}(S)$ is a direct summand of a bounded complex of trivial vector bundles over $\operatorname{Spec}(S)$; the complement $C^{\prime}$ is a strict perfect complex and can be chosen to have trivial differential. Indeed, choose $C_{n}^{\prime}$ to be a 
finitely generated projective module so that $C_{n} \oplus C_{n}^{\prime}$ is finitely generated free (with $C_{n}^{\prime}=0$ whenever $C_{n}=0$ ) and equip $C^{\prime}$ with zero-differentials.

Proposition 2.2 (Characterisations of finite domination). The following five statements are equivalent for a bounded below chain complex $C$ of projective $S$-modules:

(1) The complex $C$ is $S$-perfect.

(2) The complex $C$ is chain homotopy equivalent to a strict perfect complex.

(3) The complex $C$ is $S$-finitely dominated.

(4) There exist a strict perfect complex $D$ of $S$-modules and chain maps $r: D \longrightarrow C$ and $s: C \longrightarrow D$ such that $r \circ s \simeq \mathrm{id}_{C}$.

(5) The complex $C$ is a direct summand of an $S$-finitely dominated bounded below complex $E$.

Proof. The equivalence of (1) and (2) follows from the fact that any quasiisomorphism between bounded below complexes of projective modules is a homotopy equivalence. The equivalence of (2) and (3) has been shown by RANICKI [Ran85, Proposition 3.2 (ii)]. The equivalence of (3) and (4) follows from the observation above that every strict perfect complex is a direct summand of a bounded complex of trivial vector bundles. If (4) holds then $C$ is a direct summand of the mapping cylinder $Z$ of $s$ which is homotopy equivalent to $D$ and hence finitely dominated (the requisite projection map $Z \longrightarrow C$ is determined by the homotopy $r \circ s \simeq \mathrm{id}_{C}$ ), so that (5) holds. Conversely, given (5) there exists a strict perfect complex $D$ homotopy equivalent to $E$ (using the already known fact that (3) implies (2)). Then the composition of $C \longrightarrow E \simeq D$ and $D \simeq E \longrightarrow C$ is homotopic to $\mathrm{id}_{C}$ so that (4) holds.

\section{The Basic homological SETUP}

In the sequel we will repeatedly need the following homological constructions related to diagrams (in some category of modules) of the shape

$$
\mathcal{M}=\left(M^{-} \stackrel{\mu^{-}}{\longrightarrow} M \stackrel{\mu^{+}}{\stackrel{4}{\longrightarrow}} M^{+}\right) \text {. }
$$

(A map of such diagrams is a triple of maps $\phi=\left(f^{-}, f, f^{+}\right)$compatible with the structure maps.) - For a diagram of $S$-modules of shape (3.1), we let $H(\mathcal{M})$ denote the chain complex $M \stackrel{-\mu^{-}+\mu^{+}}{\longleftarrow} M^{-} \oplus M^{+}$concentrated in chain degrees -1 and 0 ; we write $H^{q}(\mathcal{M})$ for the $(-q)$ th homology module of $H(\mathcal{M})$ call this the $q$ th cohomology module of $\mathcal{M}$. So $H^{0}(\mathcal{M})=\operatorname{ker}\left(-\mu^{-}+\mu^{+}\right)$and $H^{1}(\mathcal{M})=\operatorname{coker}\left(-\mu^{-}+\mu^{+}\right)$, while $H^{q}(\mathcal{M})=0$ for $q \neq 0,1$. It can be shown that $H^{q}(\mathcal{M}) \cong \lim _{\leftarrow}{ }^{q}(\mathcal{M})$ for all $q$.

More generally, for a diagram of $S$-module chain complexes (3.1) we denote by $\mathbf{H}(\mathcal{M})$ the totalisation of the double complex $H(\mathcal{M})$. More explicitly, we have $\mathbf{H}(\mathcal{M})_{n}=M_{n}^{-} \oplus M_{n}^{+} \oplus M_{n+1}$ with differential given by

$$
\left(a^{-}, a, a^{+}\right) \mapsto\left(d^{-}\left(a^{-}\right), d^{+}\left(a^{+}\right),-\mu^{-}\left(a^{-}\right)+\mu^{+}\left(a^{+}\right)-d(a)\right),
$$

where $d, d^{+}$and $d^{-}$are the differentials of the chain complexes $M, M^{+}$ and $M^{-}$, respectively. The complex $\mathbf{H}(\mathcal{M})$ is called the hyper-cohomology chain complex of $\mathcal{M}$, and the $(-q)$ th homology module of $\mathbf{H}(\mathcal{M})$ is the hyper-cohomology module $\mathbf{H}^{q}(\mathcal{M})$. 
A map $\phi=\left(f^{-}, f, f^{+}\right): \mathcal{M} \longrightarrow \mathcal{N}$ of diagrams of chain complexes induces a map $\phi_{*}: \mathbf{H}(\mathcal{M}) \longrightarrow \mathbf{H}(\mathcal{N})$. We also have a chain complex inclusion $\iota: H^{0}(\mathcal{M}) \longrightarrow \mathbf{H}(\mathcal{M})$ where the source is the chain complex obtained by applying the functor $H^{0}$ to $\mathcal{M}$ in each chain level.

Lemma 3.2. If the components of $\phi$ are all quasi-isomorphisms, then $\phi_{*}$ is a quasi-isomorphism. If $H^{1}(\mathcal{M})=0$ (levelwise application of $H^{1}$ ) then the map $\iota$ is a quasi-isomorphism.

Proof. The first claim follows easily from the five lemma and the existence of a short exact sequence

$$
0 \longrightarrow M[1] \longrightarrow \mathbf{H}(\mathcal{M}) \longrightarrow M^{-} \oplus M^{+} \longrightarrow 0
$$

natural with respect to maps of diagrams $\phi$. - For the second claim, note that the hypothesis $H^{1}(\mathcal{M})=0$ translates into the sequence

$$
0 \longleftarrow M \stackrel{-\mu^{-}+\mu^{+}}{\longleftarrow} M^{-} \oplus M^{+} \longleftarrow H^{0}(\mathcal{M}) \longleftarrow 0
$$

being exact (in each chain level) so that $H^{0}(\mathcal{M})$ is quasi-isomorphic to the "homotopy fibre" of the map $-\mu^{-}+\mu^{+}$(just as $M$ is then quasi-isomorphic to the mapping cone of $\iota$ ). The complex $\mathbf{H}(\mathcal{M})$ is a model for the homotopy fibre. To give a more formal argument, the mapping cone of $\iota$ is the totalisation of the double complex (3.3) (concentrated in columns $-1,0$ and 1), which is acyclic as the double complex has exact rows.

\section{The Projective Line}

We will now define categories of modules on the projective line $\mathbb{P}^{1}$ over $R$, in the spirit of BASS [Bas68, §XII.9].

Definition 4.1 (Quasi-coherent sheaves and vector bundles).

(1) A quasi-coherent sheaf on $\mathbb{P}^{1}$, or just sheaf for short, is a diagram $\mathcal{M}$ of the form (3.1) such that

- the entries are modules over the rings $R\left[x^{-1}\right], R\left[x, x^{-1}\right]$ and $R[x]$, respectively;

- the maps $\mu^{-}$and $\mu^{+}$are $R\left[x^{-1}\right]$-linear and $R[x]$-linear, respectively;

- both adjoint maps

$$
M^{-} \otimes_{R\left[x^{-1}\right]} R\left[x, x^{-1}\right] \longrightarrow M \longleftarrow M^{+} \otimes_{R[x]} R\left[x, x^{-1}\right]
$$

are isomorphisms of $R\left[x, x^{-1}\right]$-modules.

(2) The category of quasi-coherent sheaves is denoted $\mathfrak{Q} \mathfrak{C} \mathfrak{o h}\left(\mathbb{P}^{1}\right)$. Morphisms are triples $\left(f^{-}, f, f^{+}\right)$of linear maps compatible with the structure maps.

(3) The sheaf (3.1) is a vector bundle if all its constituent modules are finitely generated projective over their respective ground rings.

It might be useful to recall here that $\mathbb{P}^{1}$ has a ZARISKI-open cover consisting of the affine lines $U^{+}=\operatorname{Spec} R[x]$ and $U^{-}=\operatorname{Spec} R\left[x^{-1}\right]$, with intersection $U^{-} \cap U^{+}=T=\operatorname{Spec} R\left[x, x^{-1}\right] \subset \mathbb{P}^{1}$. 
Definition 4.2. Given a sheaf $\mathcal{M}$ as in 4.1 and an integer $n$, we denote by $\mathcal{M}(n)$ any sheaf of the form $M^{-} \stackrel{x^{k} \mu^{-}}{\longrightarrow} M^{\stackrel{x^{-\ell} \mu^{+}}{\longleftarrow}} M^{+}$with $k, \ell \in \mathbb{Z}$ such that $k+\ell=n$ (all these sheaves are isomorphic in $\left.\mathfrak{Q} \mathfrak{C o h}\left(\mathbb{P}^{1}\right)\right)$. We call $\mathcal{M}(n)$ an $n$th twist of $\mathcal{M}$.

In the context of sheaves, we will write $H\left(\mathbb{P}^{1} ; \mathcal{M}\right)$ for $H(\mathcal{M})$ to emphasise the similarity to algebraic geometry (in fact, the modules $H^{q}\left(\mathbb{P}^{1} ; \mathcal{M}\right)$ are sheaf cohomology modules, computed from a ČECH complex, in case $R$ is a commutative ring). We similarly use the notation $\mathbf{H}\left(\mathbb{P}^{1} ; \mathcal{M}\right)$ to denote $\mathbf{H}(\mathcal{M})$ for a chain complex $\mathcal{M}$ of sheaves.

Example 4.3. The $n$th twisting sheaf, denoted $\mathcal{O}(n)$, is a vector bundle of the form

$$
R\left[x^{-1}\right] \stackrel{x^{k}}{\longrightarrow} R\left[x, x^{-1}\right] \stackrel{x^{-\ell}}{\longleftarrow} R[x]
$$

with $k, \ell \in \mathbb{Z}$ such that $k+\ell=n$; the structure maps are inclusions followed by multiplication. For $q, n \in \mathbb{Z}$ we have

$$
H^{q}\left(\mathbb{P}^{1} ; \mathcal{O}(n)\right) \cong \begin{cases}R^{n+1} & \text { for } q=0 \text { and } n \geq 0 \\ R^{-n-1} & \text { for } q=1 \text { and } n \leq-2 \\ 0 & \text { otherwise. }\end{cases}
$$

In fact, the $H^{q}\left(\mathbb{P}^{1} ; \mathcal{O}(n)\right)$ can be identified with free $R$-submodules of the $R$-module $R\left[x, x^{-1}\right]$; bases are $\left\{x^{-\ell}, x^{-\ell+1}, \cdots, x^{k}\right\}$ in the first case, and $\left\{x^{k+1}, x^{k+2}, \cdots, x^{-\ell-1}\right\}$ in the second.

Lemma 4.4 (Extending morphisms from $T$ to $\mathbb{P}^{1}$ ). Suppose we have two sheaves $\mathcal{Z}=\left(Z^{-} \stackrel{\zeta^{-}}{\longrightarrow} Z \stackrel{\zeta^{+}}{\longleftarrow} Z^{+}\right)$and $\mathcal{Y}=\left(Y^{-} \stackrel{v^{-}}{\longrightarrow} Y \stackrel{v^{+}}{\stackrel{S}{\longrightarrow}} Y^{+}\right)$on $\mathbb{P}^{1}$, and a homomorphism $f: Z=\left.\left.\mathcal{Z}\right|_{T} \longrightarrow \mathcal{Y}\right|_{T}=Y$. Suppose that $Z^{-}$and $Z^{+}$ are finitely generated, and that $v^{-}$and $v^{+}$are injective (equivalently, $Y^{ \pm}$ have no $x^{ \pm 1}$-torsion). Then there exist integers $k, \ell \geq 0$ and homomorphisms $f^{ \pm}: Z^{ \pm} \longrightarrow Y^{ \pm}$fitting into a commutative diagram

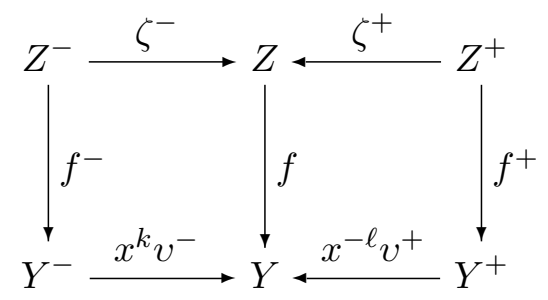

so that $f$ extends to a map of sheaves $\left(f^{-}, f, f^{+}\right): \mathcal{Z} \longrightarrow \mathcal{Y}(k+\ell)$.

Proof. Choose an epimorphism $\epsilon^{+}: F^{+} \longrightarrow Z^{+}$, with $F^{+}$finitely generated free. The composition $f \circ \zeta^{+} \circ \epsilon^{+}$factors as $F^{+} \stackrel{\phi^{+}}{\longrightarrow} Y^{+} \stackrel{x^{-\ell} v^{+}}{\longrightarrow} Y$, for some $\ell \geq 0$, as $Y \cong Y^{+} \otimes_{R[x]} R\left[x, x^{-1}\right]$ is the colimit of $Y^{+} \stackrel{x}{\longrightarrow} Y^{+} \stackrel{x}{\longrightarrow} \cdots$. Now the image of $\operatorname{ker}\left(\epsilon^{+}\right)$under $\phi^{+}$in $Y^{+}$is trivial; indeed, the map $x^{-\ell} v^{+}$ is injective, so we can check triviality by post-composing with this map. But then, by construction, we are reduced to showing that the image of $\operatorname{ker}\left(\epsilon^{+}\right)$ in $Y$ under the map $x^{-\ell} v^{+} \circ \phi^{+}=f \circ \zeta^{+} \circ \epsilon^{+}$is trivial, which is clear. It follows that $\phi^{+}$induces a map $f^{+}: Z^{+}=F^{+} / \operatorname{ker}\left(\epsilon^{+}\right) \longrightarrow Y^{+}$which, when post-composed with $x^{-\ell} v^{+}$, agrees with $f \circ \zeta^{+}$. - The construction of $f^{-}$ follows the symmetric procedure. 


\section{An algebro-geometric approach to Theorem A}

We write $R \llbracket x \rrbracket$ for the ring of formal power series in $x$; its localisation $R((x))=R \llbracket x \rrbracket_{x}$ by $x$ is the ring of formal LAURENT series in $x$. We have the variants $R \llbracket x^{-1} \rrbracket$ and $R\left(\left(x^{-1}\right)\right)$ of formal power series and formal LAURENT series in $x^{-1}$. Clearly $R((x)) \cap R\left(\left(x^{-1}\right)\right)=R\left[x, x^{-1}\right]$.

The relevant observation for us is that $N^{+}=$Spec $R \llbracket x \rrbracket$ is an infinitesimal (formal) neighbourhood of $0 \in U^{+} \subset \mathbb{P}^{1}$, and that Spec $R((x))$ corresponds to $N^{+} \backslash\{0\}$. Replacing $x$ by $x^{-1}$ gives infinitesimal neighbourhoods of $\infty \in U^{-} \subset \mathbb{P}^{1}$, with and without the point $\infty$ included.

Definition 5.1 (Strict perfect and perfect complexes). A chain complex $\mathcal{C}=\left(C^{-} \longrightarrow C \longleftarrow C^{+}\right)$of sheaves is called strict perfect if it is a bounded complex of vector bundles. We call $\mathcal{C}$ perfect if it is connected by a chain of quasi-isomorphisms in $\mathfrak{Q} \mathfrak{C o h}\left(\mathbb{P}^{1}\right)$ to a strict perfect complex. Here a quasi-isomorphism is a map of complexes of sheaves such that all three of its constituent maps of chain complexes of modules are quasi-isomorphisms.

Theorem 5.2 (Algebro-geometric reformulation of Theorem A). Let $C$ be a bounded complex of trivial vector bundles on the algebraic torus $T$. The push-forward $p_{*} C$ is an $R$-perfect complex if $C$ is homologically trivial infinitesimally near 0 and $\infty$ (that is, on $N^{+} \backslash\{0\}$ and $N^{-} \backslash\{\infty\}$ ).

We begin the proof by observing that bounded complexes of trivial vector bundles on $T$ extend to strict perfect complexes on $\mathbb{P}^{1}$ :

Proposition 5.3 (Extending complexes of trivial vector bundles). Given a bounded chain complex $C$ of trivial vector bundles on $T$, there exists a strict perfect complex $\mathcal{V}=\left(V^{-} \longrightarrow V \longleftarrow V^{+}\right)$of sheaves such that

(1) the complexes $V^{-}$and $V^{+}$consist of finitely generated free modules;

(2) the restriction $V=\left.\mathcal{V}\right|_{T}$ is isomorphic to the complex $C$;

(3) the complex of global sections $H^{0}\left(\mathbb{P}^{1} ; \mathcal{V}\right)=\lim _{\leftarrow} \mathcal{V}$ is a bounded complex of finitely generated free $R$-modules;

(4) the higher cohomology modules $H^{j}\left(\mathbb{P}^{1} ; \mathcal{V}\right)=\lim _{\leftarrow}^{j} \mathcal{V}$ are trivial for all $j \geq 1$ in each chain degree.

In fact, given a finitely generated free $R\left[x, x^{-1}\right]$-module $M$ of $\operatorname{rank}^{1} r$, the sheaf $\bigoplus_{r} \mathcal{O}(n)$, for any $n \in \mathbb{Z}$, has the property that its restriction to $T$ is isomorphic to $M$. The main point is that the differentials can be lifted to $\mathbb{P}^{1}$ as well, by downward induction on the chain degree: Assuming $\mathcal{V}_{m+1}$ has been constructed, set $\mathcal{Y}=\bigoplus_{r} \mathcal{O}(0)$ for $r$ a rank of $C_{m}$, and apply Lemma 4.4 to $\mathcal{Z}=\mathcal{V}_{m+1}$ and $f=\partial: V_{m+1} \longrightarrow V_{m} \cong\left(R\left[x, x^{-1}\right]\right)^{r}$. Finally set $\mathcal{V}_{m}=\overline{\mathcal{Y}}(k+\ell)$. Since the structure maps of $\mathcal{O}(n)$ are injective, it is easy to check that the extension of $f$ to $\mathbb{P}^{1}$ is a differential. The Proposition now follows from the calculations in Example 4.3.

Back to Theorem 5.2, let us thus extend our complex $C$ to a strict perfect complex $\mathcal{C}=\left(C^{-} \stackrel{\overline{\mu^{-}}}{\longrightarrow} C \stackrel{\mu^{+}}{\longleftarrow} C^{+}\right)$as described in Proposition 5.3. From

\footnotetext{
${ }^{1}$ By rank we mean the cardinality of some basis of $M$; this number might not be uniquely determined by $M$ unless $R$ has the invariant basis property
} 
Lemma 3.2 we know that the map $\iota: H^{0}\left(\mathbb{P}^{1} ; \mathcal{C}\right) \longrightarrow \mathbf{H}\left(\mathbb{P}^{1} ; \mathcal{C}\right)$ is a quasiisomorphism; note that the source of this map is a bounded complex of finitely generated free $R$-modules, by construction.

Observe that for $R$ commutative and NOETHERian, the rings $R \llbracket x \rrbracket$ and $R\left[x, x^{-1}\right]$ are flat over $R[x]$ so that Spec $R \llbracket x \rrbracket \amalg \operatorname{Spec} R\left[x, x^{-1}\right] \longrightarrow \operatorname{Spec} R[x]$ is a covering of the affine line in the fpqc topology; furthermore, the intersection of the two covering sets is Spec $R((x))$. So for general $R$, we represent the restriction $C^{+}=\left.\mathcal{C}\right|_{U^{+}}$of $\mathcal{C}$ to $U^{+}=\operatorname{Spec} R[x]$ in the fpqc topology by the diagram

$$
\mathcal{C}_{\mathrm{fpqc}}^{+}=\left(C^{+} \underset{R[x]}{\otimes} R \llbracket x \rrbracket \longrightarrow C^{+} \underset{R[x]}{\otimes} R((x)) \longleftarrow C^{+} \otimes_{R[x]} R\left[x, x^{-1}\right]\right) .
$$

Since $C^{+}$consists of free $R[x]$-modules, and since the diagram of $R[x]$-modules $R \llbracket x \rrbracket \longrightarrow R((x)) \longleftarrow R\left[x, x^{-1}\right]$ has $H^{0}=R[x]$ and $H^{1}=0$, the first map in the composition $\kappa^{+}$of canonical maps

$$
C^{+} \stackrel{\cong}{\longrightarrow} H_{\text {fpqc }}^{0}\left(U^{+} ; \mathcal{C}_{\text {fpqc }}^{+}\right) \stackrel{\simeq}{\longrightarrow} \mathbf{H}_{\text {fpqc }}\left(U^{+} ; \mathcal{C}\right)
$$

is an isomorphism, where the source of $\iota$ stands for the chain complex $H^{0}\left(\mathcal{C}_{\text {fpqc }}^{+}\right)$and the target for $\mathbf{H}\left(\mathcal{C}_{\text {fpqc }}^{+}\right)$. Moreover, the structure isomorphism $C^{+} \otimes_{R[x]} R\left[x, x^{-1}\right] \cong C$ of $\mathcal{C}$ results in a chain map $\lambda^{+}: \mathbf{H}_{\mathrm{fpqc}}\left(U^{+} ; \mathcal{C}\right) \longrightarrow C$ such that $\mu^{+}=\lambda^{+} \circ \kappa^{+}$.

We have an analogous quasi-isomorphism $\kappa^{-}: C^{-} \longrightarrow \mathbf{H}_{\text {fpqc }}\left(U^{-} ; \mathcal{C}\right)=$ $\mathbf{H}\left(\mathcal{C}_{\text {fpqc }}^{-}\right)$, and a factorisation $\mu^{-}=\lambda^{-} \circ \kappa^{-}: C^{-} \longrightarrow C$.

Now note that by hypothesis the chain complex

$$
C^{+} \underset{R[x]}{\otimes} R((x)) \cong C^{+} \underset{R[x]}{\otimes} R\left[x, x^{-1}\right] \underset{R\left[x, x^{-1}\right]}{\otimes} R((x)) \cong C \underset{R\left[x, x^{-1}\right]}{\otimes} R((x))
$$

is acyclic. This means we can replace the middle entry in (5.4) by the trivial chain complex; by Lemma 3.2, the resulting map

$$
\begin{aligned}
\mathbf{H}_{\mathrm{fpqc}}\left(U^{+} ; \mathcal{C}\right) \longrightarrow \mathbf{H}\left(C^{+} \otimes_{R[x]} R \llbracket x \rrbracket\right. & \longrightarrow 0 \longleftarrow C) \\
& =\left(C^{+} \otimes_{R[x]} R \llbracket x \rrbracket\right) \oplus C
\end{aligned}
$$

is a quasi-isomorphism. We have a similar quasi-isomorphism, constructed in a similar manner, $\mathbf{H}_{\text {fpqc }}\left(U^{-} ; \mathcal{C}\right) \longrightarrow\left(C^{-} \otimes_{R\left[x^{-1}\right]} R \llbracket x^{-1} \rrbracket\right) \oplus C$.

All these maps fit into the following commutative diagram:

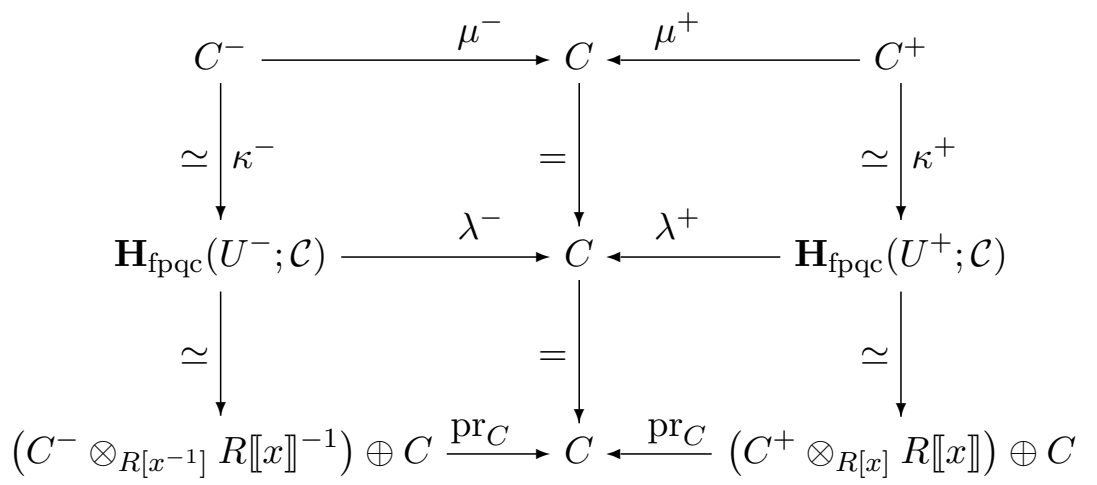

Application of $\mathbf{H}$ to each row then results in a quasi-isomorphism from $\mathbf{H}\left(\mathbb{P}^{1} ; \mathcal{C}\right)$ (the first row) with a chain complex that contains, by construction, 
the complex $\mathbf{H}(C \stackrel{=}{\longrightarrow} C \stackrel{=}{=} C)$ as a direct summand. Now the latter is quasi-isomorphic to $C$, by Lemma 3.2 , and the former is quasi-isomorphic to a bounded complex of finitely generated free $R$-modules, as noted before. It follows from Proposition 2.2 that $C$ is perfect as claimed.

\section{Extending SheAves From the torus to the PROJeCtive Line}

The proof given in the previous section relies on extending the given chain complex $C$ to a complex of vector bundles on $\mathbb{P}^{1}$. There is a $K$-theoretical obstruction to extension, as described in this section (which is motivated by, and modelled on, the discussion of extension problems by THOMASON and Trobaugh [TT90, $\S 5.5])$. As a matter of terminology, we say that a perfect complex $C$ of $R\left[x, x^{-1}\right]$-modules extends to $\mathbb{P}^{1}$ up to quasi-isomorphism if there exists a strict perfect complex $\mathcal{V}$ of sheaves on $\mathbb{P}^{1}$ such that $\left.\mathcal{V}\right|_{T}$ is quasi-isomorphic to $C$.

Lemma 6.1. Let $0 \longrightarrow C_{1} \stackrel{\alpha}{\longrightarrow} C_{2} \stackrel{\beta}{\longrightarrow} C_{3} \longrightarrow 0$ be a short exact sequence of perfect complexes of $R\left[x, x^{-1}\right]$-modules. Suppose that two of the three complexes extend to $\mathbb{P}^{1}$ up to quasi-isomorphism. Then so does the third.

Proof. Suppose first that $C_{1}$ and $C_{2}$ extend up to quasi-isomorphism. Let $\nu: C_{1} \longrightarrow C_{2}$ be any chain map; we will show that then the mapping cone of $\nu$ extends to $\mathbb{P}^{1}$ up to quasi-isomorphism. - By hypothesis we find strict perfect complexes $\mathcal{V}_{1}$ and $\mathcal{V}_{2}$ on $\mathbb{P}^{1}$ with $\left.\mathcal{V}_{1}\right|_{T} \simeq C_{1}$ and $\left.\mathcal{V}_{2}\right|_{T} \simeq C_{2}$. Together with $\nu$ these quasi-isomorphisms determine a morphism $\left.\left.\mathcal{V}_{1}\right|_{T} \longrightarrow \mathcal{V}_{2}\right|_{T}$ in the derived category of $R\left[x, x^{-1}\right]$. Since $\left.\mathcal{V}_{1}\right|_{T}$ is strict perfect, we can represent this morphism by an actual chain map $\omega$. Note that the mapping cone of $\omega$ is quasi-isomorphic to the mapping cone of $\nu$.

After replacing $\mathcal{V}_{2}$ by a twist $\mathcal{V}_{2}(k)$ for sufficiently large $k>0$, we can extend $\omega$ to a map $\Omega$ of complexes of sheaves on $\mathbb{P}^{1}$, which satisfies $\left.\Omega\right|_{T}=\omega$; this follows from Lemma 4.4, applied to $\mathcal{Z}=\mathcal{V}_{1}, \mathcal{Y}=\mathcal{V}_{2}$ and $f=\omega$ in each chain level (the constructed extensions are automatically compatible with the differentials). The mapping cone of $\Omega$ is then a strict perfect complex of sheaves on $\mathbb{P}^{1}$ which restricts to the mapping cone of $\omega$ on $T$, thus extending the mapping cone of $\nu$ up to quasi-isomorphism.

The theorem now follows easily in view of the quasi-isomorphisms $C_{3} \simeq$ Cone $(\alpha), C_{1} \simeq \operatorname{cone}(\beta)[-1]$ and $C_{2} \simeq \operatorname{cone}\left(\operatorname{cone}(\alpha) \longrightarrow C_{1}[1]\right)[-1]$.

Let $K_{0}(S)$ denote the GROTHENDIECK group of isomorphism classes of finitely generated projective $S$-modules. That is, $K_{0}(S)$ is the quotient of the free ABELian group generated by the isomorphism classes $[P]$, for $P$ a finitely generated projective $S$-module, by the subgroup with one generator $[P]+[R]-[Q]$ for every short exact sequence

$$
0 \longrightarrow P \longrightarrow Q \longrightarrow R \longrightarrow 0
$$

of finitely generated projective $S$-modules. It follows from general $K$-theory, specifically from 1.5.6, 1.11.2 and 1.11.7 of [TT90], that we could have defined $K_{0}(A)$ as the quotient of the free ABELian group generated by quasiisomorphism classes of strict perfect $S$-module complexes by the subgroup 
with one generator $[P]+[R]-[Q]$ for each short exact sequence (6.2) of strict perfect complexes which splits in each chain level.

By replacing " $S$-module" by "sheaf on $\mathbb{P}^{1}$ ", and "finitely generated projective $S$-module" by "vector bundle on $\mathbb{P}^{1}$ " we obtain two equivalent definitions of $K_{0}\left(\mathbb{P}^{1}\right)$.

It might be worthwhile to point out that in the case of $S$-modules, it is automatic that the sequence (6.2) splits, and that in the case of strict perfect complexes of $S$-modules the sequence (6.2) splits in each chain level. Also, two strict perfect complexes of $S$-modules are quasi-isomorphic if and only if they are chain homotopy equivalent, so we could have replaced "quasiisomorphism class" by "homotopy class". The situation is entirely different in the case of sheaves on $\mathbb{P}^{1}$ where, in general, a sequence (6.2) will be nonsplit, and where quasi-isomorphism does not imply homotopy equivalence.

Theorem 6.3 (Precise formulation of Theorem B). Let $C$ be a strict perfect complex of $R\left[x, x^{-1}\right]$-modules. The following three statements are equivalent:

(1) The complex $C$ extends to $\mathbb{P}^{1}$ up to quasi-isomorphism.

(2) The class $[C] \in K_{0}\left(R\left[x, x^{-1}\right]\right)$ lies in the image of the pullback map

$$
p^{*}: K_{0}(R) \longrightarrow K_{0}\left(R\left[x, x^{-1}\right]\right), \quad[M] \mapsto\left[M \otimes_{R} R\left[x, x^{-1}\right]\right]
$$

which is induced by the map $p: T \longrightarrow P$.

(3) The class $[C] \in K_{0}\left(R\left[x, x^{-1}\right]\right)$ lies in the image of the restriction map

$$
i^{*}: K_{0}\left(\mathbb{P}^{1}\right) \longrightarrow K_{0}\left(R\left[x, x^{-1}\right]\right), \quad\left[C^{-} \longrightarrow C \longleftarrow C^{+}\right] \mapsto[C]
$$

which is induced by the inclusion map $i: T \subset \mathbb{P}^{1}$.

Proof. We show first that statements (1) and (3) are equivalent. The implication $(1) \Rightarrow(3)$ is trivial.

A bounded complex of finitely generated free $R\left[x, x^{-1}\right]$-modules extends to $\mathbb{P}^{1}$, as observed in Proposition 5.3. Next, for $C$ an arbitrary strict perfect complex we can find a bounded complex $C^{\prime}$ of finitely generated projective $R\left[x, x^{-1}\right]$-modules such that $C \oplus C^{\prime}$ consists of finitely generated free modules, and thus extends to $\mathbb{P}^{1}$.

Now let $\Pi$ denote free ABELian group generated by the quasi-isomorphism classes $\langle A\rangle$ of strict perfect complexes $A$ of $R\left[x, x^{-1}\right]$-modules, and let $\Lambda$ be the subgroup generated by the relations

$$
\langle A\rangle+\langle B\rangle-\langle A \oplus B\rangle,
$$

$\langle K\rangle$ for $K$ extending to $\mathbb{P}^{1}$ up to quasi-isomorphism.

Define $\pi=\Pi / \Lambda$; we denote the image of $\langle C\rangle$ in $\pi$ by $[C]$. For $C$ and $C^{\prime}$ as in the previous paragraph the relations clearly imply $[C]+\left[C^{\prime}\right]=0$ so that $C^{\prime}$ represents in inverse of $[C]$.

Now suppose that $C$ is such that $[C]=0$ in $\pi=\Pi / \Lambda$. Then we must have $\langle C\rangle \in \Lambda$, that is, we find finitely many $A_{i}, B_{i}, \bar{A}_{j}$ and $\bar{B}_{j}$, and finitely 
many $K_{k}$ and $L_{\ell}$ that extend to $\mathbb{P}^{1}$ up to quasi-isomorphism, such that

$$
\begin{aligned}
\langle C\rangle+\sum_{j}\left(\left\langle\bar{A}_{j}\right\rangle+\left\langle\bar{B}_{j}\right\rangle-\left\langle\bar{A}_{j} \oplus \bar{B}_{j}\right\rangle\right)+\sum_{\ell}\left\langle L_{\ell}\right\rangle & \\
= & \sum_{i}\left(\left\langle A_{i}\right\rangle+\left\langle B_{i}\right\rangle-\left\langle A_{i} \oplus B_{i}\right\rangle\right)+\sum_{k}\left\langle K_{k}\right\rangle .
\end{aligned}
$$

That is, we have the equality

$$
\begin{aligned}
\langle C\rangle+\sum_{j}\left(\left\langle\bar{A}_{j}\right\rangle+\left\langle\bar{B}_{j}\right\rangle\right) & +\sum_{i}\left\langle A_{i} \oplus B_{i}\right\rangle+\sum_{\ell}\left\langle L_{\ell}\right\rangle \\
& =\sum_{i}\left(\left\langle A_{i}\right\rangle+\left\langle B_{i}\right\rangle\right)+\sum_{j}\left\langle\bar{A}_{j} \oplus \bar{B}_{j}\right\rangle+\sum_{j}\left\langle K_{j}\right\rangle
\end{aligned}
$$

in the free ABELian group $\Pi$; this implies that the quasi-isomorphism classes occurring on both sides must agree, counted with multiplicities. That is, we have a quasi-isomorphism $C \oplus A \oplus L \simeq A \oplus K$ where we write $A=$ $\bigoplus_{i}\left(A_{i} \oplus B_{i}\right) \oplus \bigoplus_{j}\left(\bar{A}_{j} \oplus \bar{B}_{j}\right), L=\bigoplus_{\ell} L_{\ell}$ and $K=\bigoplus_{k} K_{k}$. Let $A^{\prime}$ represent an inverse for $A$ in $\pi$ as in the first paragraph of this proof; then $A^{\prime} \oplus A$ extends to $\mathbb{P}^{1}$ up to quasi-isomorphism so that $A^{\prime} \oplus A \oplus L$ and $C \oplus A^{\prime} \oplus A \oplus L \simeq$ $A^{\prime} \oplus A \oplus K$ do too. It follows from Lemma $\underline{6.1}$ that $C$ extends to $\mathbb{P}^{1}$ up to quasi-isomorphism.

We now know that $[C]=0 \in \pi$ if and only if $C$ extends to $\mathbb{P}^{1}$ up to quasi-isomorphism. It remains to observe that $\pi=\operatorname{coker}\left(i^{*}\right)$. This is almost immediate from the presentation of $K$-groups given at the beginning of this section; the one discrepancy we have is that $\pi$ incorporates the relation (6.4a) for split short exact sequences only, whereas in $K_{0}\left(R\left[x, x^{-1}\right]\right)$ we have a relation for all levelwise split sequences. It is enough to verify the following assertion: Given a short exact sequence $0 \longrightarrow C \stackrel{f}{\longrightarrow} D \stackrel{g}{\longrightarrow} E \longrightarrow 0$ of strict perfect complexes of $R\left[x, x^{-1}\right]$-modules, we have $[C]+[E]=[D]$ in the group $\pi$. For this, let $C^{\prime}$ and $E^{\prime}$ represent inverses of $[C]$ and $[E]$ in $\pi$, as in the beginning of this proof. Then we have a short exact sequence

$$
0 \longrightarrow C \oplus C^{\prime} \stackrel{\left(f, \mathrm{id}_{C^{\prime}}, 0\right)}{\longrightarrow} D \oplus C^{\prime} \oplus E^{\prime} \stackrel{\left(g, \mathrm{id}_{E^{\prime}}\right)}{\longrightarrow} E \oplus E^{\prime} \longrightarrow 0
$$

of strict perfect complexes, with first and third term being free; it follows that $D \oplus C^{\prime} \oplus E^{\prime}$ extends to $\mathbb{P}^{1}$ up to quasi-isomorphism (Lemma 6.1) and thus represents $0 \in \pi$. Consequently, $[D]=-\left[C^{\prime}\right]-\left[E^{\prime}\right]=[C]+[E] \in \pi$.

Finally, we need to prove that statements (2) and (3) are equivalent. As shown by BAss [Bas68, §XII.9] and QuILlen [Qui73, §8.3] there is an isomorphism $K_{0}(R) \times K_{0}(R) \stackrel{\cong}{\longrightarrow} K_{0}\left(\mathbb{P}^{1}\right)$ which sends the element $([M],[N])$ to $\left[M \otimes_{R} \mathcal{O}(0)\right]+\left[M \otimes_{R} \mathcal{O}(-1)\right]$, which is mapped by $i^{*}$ to $p^{*}([M])+p^{*}([N])$. It follows that the image of $i^{*}$ coincides with the image of the map $p^{*}$.

The map $p^{*}$ exhibits $K_{0}(R)$ as a direct summand of $K_{0}\left(R\left[x, x^{-1}\right]\right)$ with complement $\operatorname{coker}\left(p^{*}\right) \cong K_{-1}(R) \oplus N K_{0}(R) \oplus N K_{0}(R)$, according to the BASs-Heller-Swan formula [Bas68, Corollary XII.7.6]. Thus by Theorem 6.3, the group $K_{-1}(R) \oplus N K_{0}(R) \oplus N K_{0}(R)$ is trivial if and only if every strict perfect complex of $R\left[x, x^{-1}\right]$-modules extends to $\mathbb{P}^{1}$ up to quasiisomorphism. For the truncated polynomial ring $R=\mathbb{Z}[T] / T^{m}$, where 
$m \geq 2$ is not a prime power, $K_{-1}(R) \neq\{0\}$ [Bas68, Theorem XII.10.6 (b)]; it follows that $p^{*}$ is not surjective in this case so that there exist strict perfect complexes on $T$ which do not extend to $\mathbb{P}^{1}$ up to quasi-isomorphism.

The previous paragraph implies that we cannot simply ignore the freeness assumption in Theorem A as the proof breaks down at the very first step. It thus comes as a surprise that the trivial observation made at the beginning of the paper, that every strict perfect complex can be complemented so that the resulting complex consists of free modules, can be used to overcome this issue. As there is no (obvious) way to control the homological behaviour of the complement near 0 and $\infty$, a carefully re-phrased argument, as documented in [HQ13], is needed to generalise Theorem A from free to strict perfect $R\left[x, x^{-1}\right]$-module complexes.

\section{REFERENCES}

[Bas68] Hyman Bass, Algebraic K-theory, W. A. Benjamin, Inc., New York-Amsterdam, 1968.

[HQ13] Thomas Hüttemann and David Quinn, Finite domination and Novikov rings. Iterative approach, Glasg. Math. J. 55 (2013), no. 1, pp. 145-160.

[Hüt11] Thomas Hüttemann, Double complexes and vanishing of Novikov cohomology, Serdica Math. J. 37 (2011), no. 4, pp. 295-304.

[Qui73] Daniel Quillen, Higher algebraic K-theory. I, Algebraic K-theory, I: Higher $K$-theories (Proc. Conf., Battelle Memorial Inst., Seattle, Wash., 1972), Lecture Notes in Math., vol. 341, Springer, Berlin, 1973, pp. 85-147.

[Ran85] Andrew Ranicki, The algebraic theory of finiteness obstruction, Math. Scand. 57 (1985), no. 1, pp. 105-126.

[Ran95] _ Finite domination and Novikov rings, Topology 34 (1995), no. 3, pp. 619-632.

[TT90] R. W. Thomason and Thomas Trobaugh, Higher algebraic K-theory of schemes and of derived categories, The Grothendieck Festschrift, Vol. III, Progr. Math., vol. 88, Birkhäuser Boston, Boston, MA, 1990, pp. 247-435.

Thomas Hüttemann, Queen's University Belfast, School of Mathematics and Physics, Pure Mathematics Research Centre, Belfast Bt7 1NN, UK

E-mail address: t.huettemann@qub.ac.uk

$U R L:$ http://huettemann.zzl.org/ 